\title{
Spenderbindung - Behavioristische Einflussgrößen und Implikationen für Nonprofit-Organisationen
}

Faktoren der Aufrechterhaltung der finanziellen Unterstützung; Konstrukte; Nonprofit-Organisationen; Spenderloyalität; Strukturgleichungsmodell; verhaltenswissenschaftliche Determinanten

Aufgrund verschiedener Entwicklungen auf dem Spendenmarkt steigt die Notwendigkeit an theoretisch fundierten und empirisch belegten Ausführungen über das Spenderverhalten. Dieser Beitrag betrachtet die Spenderbindung aus Spendersicht. Es wird eine begriffliche und definitorische Konkretisierung behavioristischer Antezedenz-Variablen vorgenommen und ihre Beziehungen untereinander in Zusammenhang gestellt. Bei den Determinanten handelt es sich um Commitment, Vertrauen, Zufriedenheit und Involvement. Die empirische Analyse zeigt, dass alle Determinanten einen Einfluss auf die Spenderbindung haben, wenn auch z. T. nur indirekt.

\section{Einführung}

\section{Problemhintergrund - Entwicklungen auf dem Spendenmarkt}

Seit Mitte der neunziger Jahre stagniert das Spendenaufkommen in Deutschland (TNS Infratest 2009, S. 10). Ausnahmen dieses Trends bilden lediglich Jahre mit großen Katastrophen, wie z. B. der Tsunami 2005 und das Erdbeben in Haiti 2010. Einem gleichbleibenden Spendenaufkommen steht eine wachsende Zahl von Nonprofit-Organisationen (im Folgenden: NPOs) gegenüber, die um Gelder werben. Zusätzlich zu Neugründungen drängen ausländische NPOs auf den deutschen Spendenmarkt, wie z. B. Oxfam. Der Wettbewerbsdruck ist zudem größer geworden, weil sich der Dritte Sektor zunehmend professionalisiert (Helmig/Purtschert/Beccarelli 2006, S. 353). Die Konkurrenz der NPOs zeigt sich durch eine neue Aggressivität bei der Spenderakquisition: So gab es im November 2006 mehr als 15 NPOs in Deutschland, die jeweils über eine Million Mailings verschickten (Hermes 2007, S. 101). Es herrscht damit ein Verdrängungswettbewerb im Bereich des Spendensammelns, der zu sinkenden Spendeneinnahmen pro Organisation bzw. gestiegenen Kosten für die Neuspendergewinnung führt.

Zudem lässt sich ein Wandel im Verhältnis von Staat und Bürgern hinsichtlich gesellschaftlicher und individueller Verantwortung in Deutschland feststellen. In den letzten Jahren äußerte sich dies in einem Rückgang staatlicher Unterstützungen, Kürzungen öffentlich finanzierter Sozialleistungen und einer gleichzeitigen Auslagerung an NPOs (Burgy 2008, S. 9). Eine größere Nachfrage für die Leistungen der NPOs sowie die Notwendigkeit der finanziellen Unabhängigkeit vom Staat erhöht damit die Bedeutung von Privatspenden. 
In Anbetracht dieser Entwicklungen lässt sich festhalten, dass der Druck auf die NPOs zunimmt. Für viele NPOs ist es erforderlich, umzudenken und nach Differenzierungspotenzialen im Wettbewerb sowie neuen Ausschöpfungspotenzialen des Spendenmarktes zu suchen. Unter den heutigen Bedingungen können NPOs ihre Spendeneinnahmen nicht mehr allein dadurch steigern, dass sie neue Spender werben; vielmehr gewinnen ein dauerhafter Spenderstamm und seine Bindung an Bedeutung (Urselmann 2007, S. 34).

\section{Relevanz einer Untersuchung der Spenderbindung aus Spendersicht}

Die Gründe, warum Spender ihre Unterstützung aufrechterhalten, haben für NPOs eine zentrale Bedeutung. Die Beantwortung der Frage nach den Einflussgrößen der Spenderbindung ist für die Erarbeitung effizienter und effektiver Strategien zur Förderung der Spenderbindung erforderlich. Diese Kenntnis und ihre Anwendung in spezifischen Maßnahmen der Spenderbindung scheinen aber bislang nicht vorzuliegen, denn die Bindung der Spender ist für viele NPOs schwierig: Bis zu $50 \%$ der Erstspender einer NPO unterstützen die Organisation nicht erneut; bei Mehrfachspendern sind Verlustraten von bis zu 30 \% jährlich nicht unüblich. Damit verliert eine NPO im Laufe von nur fünf Jahren 90 \% der im Jahr Null akquirierten Spender (Sargeant 2008, S. 3).

Aufgrund dieses Praxisproblems steigt die Notwendigkeit an theoretisch und empirisch fundierten Erkenntnissen über das Spenderverhalten und die Spenderbindung (Voss 2007, S. 287). Die Forschungslücke besteht in der mangelnden Kenntnis über das Spenderverhalten und die entsprechenden Einflussgrößen. Durch die Analyse der verhaltenswissenschaftlichen Determinanten der Spenderbindung trägt diese Untersuchung dazu bei, diese Schwäche zu überwinden. Betrachtet man die wissenschaftliche Auseinandersetzung mit dem Konstrukt Spenderbindung, so lässt sich feststellen, dass nur wenige empirische Untersuchungen existieren, die mit Spenderbindung explizit den hier beschriebenen Forschungsgegenstand behandeln (Sargeant 2008, S. 3). Untersuchungen fokussieren sich eher auf Mitglied- oder Patenschaften (Kristoffersen/Singh 2004; Bhattacharya/Hayagreeva/Glynn 1995). Ansonsten stehen Motive der Spendergewinnung im Vordergrund, die sich aber deutlich von der Motivation, die finanzielle Unterstützung aufrechtzuerhalten, unterscheiden (Sargeant 2008, S. 2; Bussel/Forbes 2006, S. 154; Zimmer/Nährlich 1993, S. 352 f.).

Spenderbindungsmaßnahmen lassen sich nur dann erfolgreich umsetzen, wenn sie die Anforderungen der Spender hinsichtlich der Beziehung erfüllen und die NPOs Kenntnisse darüber haben, welche Einflussgrößen die Spenderloyalität beeinflussen (Shabbir/Palihawadana/Thwaites 2007, S. 289). Somit ist es notwendig, die Sicht der Spender einzunehmen. Diese einführenden Bemerkungen verdeutlichen die Relevanz einer umfassenden wissenschaftlichen Auseinandersetzung mit der Bindung aus Spendersicht. Dieser Beitrag hilft, die Spender, ihre Erwartungen, ihre Bedürfnisse und ihr Verhalten im Rahmen längerfristiger Beziehungen zur NPO besser zu verstehen. Dementsprechend ist es das Ziel des vorliegenden Beitrags, die Konsequenzen, aber insbesondere auch verhaltenswissenschaftliche Determinanten der Spenderbindung darzustellen und in einen theoretischen Bezugsrahmen einzubinden, d. h. ihre Interdependenzen zu analysieren, sowie eine empirische Validierung vorzunehmen. Hierzu werden zunächst der Begriff Spenderbindung und die verhaltenswissenschaftlichen Determinanten definiert und die Dimensionen der Konstrukte 
bestimmt. Anschließend werden die Beziehungen und Zusammenhänge herausgearbeitet. Das dritte Kapitel beschreibt die empirische Untersuchung der zuvor theoretisch hergeleiteten Wirkungsbeziehungen, bevor abschließend eine Interpretation der Ergebnisse erfolgt und Empfehlungen für die Praxis gegeben werden.

\section{Theoretische Analyse der Spenderbindung}

\section{Spenderbindung als Zielkonstrukt der Analyse}

\section{a) Explikation des Begriffs Spenderbindung und inhaltliche Präzisierung}

Das Konstrukt Spenderbindung stellt die zu erklärende Zielgröße dar. Im kommerziellen Marketing versteht man unter dem verwandten Begriff Kundenbindung den Aufbau und die Aufrechterhaltung einer langfristigen Geschäftsbeziehung, die sich durch eine nicht zufällige Folge von Markttransaktionen zwischen Anbieter und Nachfrager auszeichnet (Krafft 2007, S. 29). Dies lässt sich auf die Spenderbindung übertragen:

Spenderbindung liegt vor, wenn innerhalb eines definierten Zeitraums eine Einzelperson (= ein Spender) freiwillig wiederholt im Sinne der NPO gehandelt hat (ex post-Betrachtung) und beabsichtigt, dies in Zukunft fortzusetzen (ex ante-Betrachtung).

Die in dieser Definition enthaltene Mehrdimensionalität ist allgemein anerkannt (Eggert/Helm/ Garnefeld 2007, S. 235). Da eine ausschließliche Fokussierung auf das bisherige Verhalten (ex post-Betrachtung) keine hinreichende Erklärung liefert, gilt es insbesondere auch die Einstellungen des Spenders als Voraussetzung der Loyalität abzufragen (Burgy 2008, S. 27). Während sich das bisherige Verhalten beobachten lässt, konzeptualisiert man Einstellungen über die Verhaltensabsichten (ex ante-Betrachtung) (Homburg/Kebbel 2001, S. 46). Die Absichten eröffnen Einsichten in die Beständigkeit der Beziehung. Deshalb operationalisiert dieser Beitrag die Bindung analog zu anderen empirischen Studien (z. B. Jaritz 2008; Neumann 2007; Sargeant/Woodliffe 2007) ausschließlich über das intentionale Verhalten.

\section{b) Begründung der Auswahl der Determinanten der Spenderbindung}

Im Vordergrund der Analyse stehen beziehungsorientierte vorökonomische Einflussgrößen (Bruhn 1999, S. 194). Andere Determinanten des Spenderverhaltens (z. B. Lebensstil) bleiben unberücksichtigt, da sie eine Gegebenheit für NPOs darstellen und keine Implikationen auf den Instrumente-Einsatz erlauben. Diese Vorgehensweise erklärt sich durch die Unterscheidung von allgemeinen Persönlichkeitsmerkmalen und produktspezifischen Kriterien als Bestimmungsgrößen des Verhaltens. Persönlichkeitsmerkmale stellen Determinanten des Verhaltens dar, die die NPO nicht beeinflussen kann (Bennett/Ali-Choudhury 2009, S. 164 f.). Die produktspezifischen Kriterien stehen hingegen in direktem Zusammenhang zur NPO und lassen sich beeinflussen. Da in diesem Beitrag jedoch keine Produkte im Fokus stehen, sondern die NPO als Organi- 
sation, sollen diese abweichend als organisationsspezifische Kriterien bezeichnet werden; sie stehen im Fokus der weiteren Betrachtung.

Behavioristische, organisationsspezifische Determinanten der Kundenbindung wurden im kommerziellen Bereich bereits stark erforscht. Mittlerweile haben sich zentrale Konstrukte herauskristallisiert: Commitment, Vertrauen und Zufriedenheit (Bruhn 2009, S. 69; Sargeant 2008, S. 3). Darüber hinaus bezieht diese Untersuchung das Involvement des Spenders ein. Bisher haben nur wenige Forscher (Jeker 2002, S. 22 ff.; Eggert 1999, S. 49 ff.) Involvement als zentralen Einflussfaktor im Bereich der Bindung untersucht; wie die Ausführungen in Abschnitt 5 zeigen, ist das Involvement aber ebenso maßgeblich für die Beziehung des Spenders zur NPO.

Die in Kapitel I 2 beschriebene Forschungslücke zum Thema Spenderbindung bezieht sich insbesondere auch auf die vier im Folgenden dargestellten behavioristischen Determinanten. Bis auf einzelne Beiträge wurden diese bisher nur mangelhaft erforscht. Dies verdeutlicht Abb. 1, welche die in den jeweiligen Studien untersuchten Einflussgrößen durch ein , $\checkmark$ “ kennzeichnet.

\begin{tabular}{|c|c|c|c|c|}
\hline $\begin{array}{l}\text { Commitment } \\
\text { des Spenders }\end{array}$ & $\begin{array}{l}\text { Spender- } \\
\text { vertrauen }\end{array}$ & $\begin{array}{c}\text { Spender- } \\
\text { zufriedenheit }\end{array}$ & $\begin{array}{c}\text { Spender- } \\
\text { involvement }\end{array}$ & Autoren \\
\hline & & $\checkmark$ & & Michalski/Helmig (2010) \\
\hline & & $\checkmark$ & & Burgy (2008) \\
\hline$\checkmark$ & $\checkmark$ & $\checkmark$ & & Sargeant (2008) \\
\hline$\checkmark$ & $\checkmark$ & & & Sargeant/Woodliffe (2007) \\
\hline \multirow[t]{3}{*}{$\checkmark$} & $\checkmark$ & $\checkmark$ & & Shabbir/Palihawadana/Thwaites (2007) \\
\hline & $\checkmark$ & $\checkmark$ & & Swanson/Davis/Zhao (2007) \\
\hline & & & $\bar{\checkmark}$ & Bennett (2006) \\
\hline$\checkmark$ & $\checkmark$ & & & Sargeant/Ford/West (2006) \\
\hline$\checkmark$ & $\checkmark$ & & & Bennett/Barkensjo (2005) \\
\hline$\checkmark$ & $\checkmark$ & & & MacMillan u. a. (2005) \\
\hline$\checkmark$ & & $\checkmark$ & & Tidwell (2005) \\
\hline$\checkmark$ & $\checkmark$ & & & Sargeant/Lee (2004) \\
\hline \multirow[t]{2}{*}{$\checkmark$} & $\checkmark$ & & & Sargeant/Lee (2004a) \\
\hline & & $\checkmark$ & & Arnett/German/Hunt (2003) \\
\hline \multirow[t]{4}{*}{$\checkmark$} & & $\checkmark$ & & Peltier/Schibrowsky/Schultz (2002) \\
\hline & $\checkmark$ & $\checkmark$ & & Sargeant/Lee (2002) \\
\hline & $\checkmark$ & $\bar{\checkmark}$ & & Sargeant/Lee (2002a) \\
\hline & $\checkmark$ & $\checkmark$ & $\checkmark$ & Thomas/Cunningham/Williams (2002) \\
\hline \multirow[t]{4}{*}{$\checkmark$} & $\checkmark$ & $\checkmark$ & & Johnson/Garbarino (2001) \\
\hline & & $\checkmark$ & & Sargeant (2001) \\
\hline & & $\checkmark$ & & Urselmann (1998) \\
\hline & & & $\bar{\checkmark}$ & Schneider (1997) \\
\hline
\end{tabular}

Abb. 1: Forschungslücke

Quelle: Eigene Darstellung

Es fällt auf, dass keine Studie sämtliche Determinanten abdeckt und damit ein umfassendes Bild vermittelt. Diese Lücke schließt der vorliegende Artikel. 


\section{Commitment des Spenders}

\section{a) Explikation des Begriffs Commitment des Spenders}

In der Literatur wird Commitment als unmittelbarste Einflussgröße der Kundenbindung angesehen (Neumann 2007, S. 157; Saab 2007, S. 2). Commitment lässt sich als empfundene Verbundenheit mit bzw. innere Verpflichtung gegenüber der anderen Beziehungspartei ansehen, die sich in dem andauernden Wunsch und Willen äußert, die Beziehung aufrechtzuerhalten (von Stenglin 2008, S. 9). Dies kann so weit gehen, dass kurzfristig Nachteile und Opfer hingenommen werden (Gundlach/Achrol/Mentzer 1995, S. 78). Dementsprechend ist Commitment des Spenders wie folgt zu definieren:

Commitment des Spenders ist seine psychisch bedingte Verbundenheit mit bzw. Verpflichtung gegenüber der von ihm unterstützten NPO, die sich in seiner Wertschätzung der Beziehung und seinem nachhaltigen Willen, sich für die Kontinuität der Beziehung mit der NPO anzustrengen, äußert.

Auch wenn die inhaltliche Präzisierung des Begriffs Commitment stellenweise Unterschiede aufweist, hat sich in der Forschung des kommerziellen Kundenbindungsmanagement ein Ansatz durchgesetzt, bei dem sich drei Dimensionen des Commitment unterscheiden lassen (Gundlach/ Achrol/Mentzer 1995, S. 79): das normative, das kalkulative und das affektive Commitment.

Normatives Commitment ist das Gefühl der Verpflichtung und Verantwortung (von Stenglin 2008, S. 14; Allen/Meyer 1990, S. 1). Dies kann durch eine persönliche Beziehung zu Mitarbeitern der Organisation entstehen, die durch den Abbruch der Beziehung geschädigt würden, oder durch eigene und gesellschaftliche Vorstellungen von Moral und Loyalität. Im Rahmen der SpenderNPO-Beziehung stellt das normative Commitment einen wichtigen Aspekt dar, denn dieser befürchtet, dass finanzielle Einbußen durch die Beendigung seiner Unterstützung eine negative Konsequenz zur Folge haben. Das Spendenmotiv besteht damit häufig in moralischen Gewissensbissen und Pflichterfüllung (Saab 2007, S. 51; Jeker 2002, S. 116).

Kalkulatives Commitment stellt eine rationale und „gefühlskalte“ Einstellung des Spenders zur NPO dar. Zur Aufrechterhaltung der Beziehung muss für den Spender ein Nettoanreiz gegeben sein, der auf einer rationalen Kosten-Nutzen-Abwägung basiert (Saab 2007, S. 71). Die Kosten spielen im Gegensatz zu einem kommerziellen Kunden eine untergeordnete Rolle. Der Spender hat keine ökonomischen Gründe, die Beziehung zur NPO einzugehen, da er nicht davon ausgehen kann, einen direkten materiellen Gegenwert für sich zu erzielen (Urselmann 1998, S. 11 f.). Obwohl der Spender keine äquivalente materielle Gegenleistung erhält, kann er aber einen persönlichen Nutzen ziehen, ohne den der Austausch nicht zustande käme (Burgy 2008, S. 62). Der Nutzen des Spenders (seine Gratifikation) entspricht hierbei der empfundenen Vorteilhaftigkeit der langfristigen finanziellen Unterstützung. Sie hat überwiegend einen immateriellen (psychologischen) Charakter, kann aber, wie im Fall der Steuerersparnis, auch materielle Aspekte aufweisen (Green/Webb 1997, S. 25).

Affektives Commitment ist das Gefühl der inneren, psychologischen Verbundenheit, welches auf einer Sympathie des Spenders für die NPO basiert. Der Spender interessiert sich für die Entwicklung der Organisation und fühlt sich persönlich betroffen. Diese Wertschätzung mündet in einer 
Identifikation mit der NPO und ihren Leistungen. Der Spender empfindet Stolz für die Beziehung, tritt als Fürsprecher auf und ergreift Partei gegenüber Kritikern (Hofmeyr/Rice 2002, S. 8 f.).

Insgesamt weisen Spender somit Commitment für die von ihnen unterstützte NPO auf, weil sie über ihr Spenden denken, sie sollten es tun (normatives Commitment), weil sie es bedürfen (kalkulatives Commitment) und/oder weil sie es wollen (affektives Commitment) (Allen/Meyer 1990, S. 3).

\section{b) Commitment als Antezedenz der Spenderbindung}

Commitment stellt laut der Literatur die zentrale Einflussgröße der Spenderbindung dar, da es die Basis für die Bindung und damit die Bereitschaft schafft, die Austauschbeziehung fortzuführen (Neumann 2007, S. 160; Tidwell 2005, S. 453). Je stärker sich ein Spender mit einer NPO verbunden bzw. ihr gegenüber verpflichtet fühlt, umso höher ist die Spenderbindung (Bhattacharya/ Hayagreeva/Glynn 1995, S. 47). Das Commitment hat einen unmittelbaren Einfluss, woraus sich die erste Hypothese ergibt:

H1: Das Commitment eines Spenders hat einen positiven Effekt auf die Spenderbindung.

\section{Vertrauen des Spenders}

\section{a) Explikation des Begriffs Vertrauen des Spenders}

Vertrauen ist eine unilaterale Erwartungs-Einstellung eines Vertrauensgebers, d. h. Vertrauenden (z. B. Spender), die auf einen Vertrauensnehmer, d. h. demjenigen, dem vertraut wird (z. B. anderes Individuum oder eine Organisation), gerichtet ist. Wenn jemand einem Vertrauensnehmer vertraut, dann bedeutet dies, dass er sich auf ihn verlässt und dessen Intentionen nicht kontrolliert (Ripperger 2003, S. 45). Vertrauen existiert nur, wenn der Vertrauensgeber wohlwollendes Verhalten und spezifische positive Eigenschaften des Vertrauensnehmers erwartet (Ranaweera/Prabhu 2003, S. 85). Vertrauen umfasst also nicht nur die Fähigkeit, ein Versprechen zu halten, sondern auch den Willen, freiwillig auf opportunistisches Verhalten zu verzichten (Ripperger 2003, S. 45). Hieraus ergeben sich Zutrauen und Verlass als Dimensionen.

Zutrauen bezeichnet die Zuversicht einer Beziehungspartei hinsichtlich der Leistungsfähigkeit der anderen Beziehungspartei, d. h. im vorliegenden Fall der NPO (Neumann 2007, S. 78). Das Zutrauen des Spenders basiert somit auf der von ihm wahrgenommenen Kompetenz der NPO (Sargeant 2008, S. 9). Verlass beschreibt die Erwartung des Vertrauensgebers hinsichtlich der Verlässlichkeit der anderen Beziehungspartei (Sirdeshmukh/Singh/Sabol 2002, S. 17). Der Spender verlässt sich auf die NPO, wenn er sie als glaubwürdig und wohlwollend wahrnimmt. Der Spender schließt also die Möglichkeit aus, dass die NPO sich opportunistisch verhält (Morgan/ Hunt 1994, S. 26). Aufbauend auf diesen zwei Dimensionen lässt sich Vertrauen wie folgt definieren: 
Spendervertrauen ist eine auf Zutrauen und Verlass basierende Einstellung des Spenders gegenüber der von ihm unterstützten NPO, dass diese trotz seiner fehlenden Kontrolle seine zukunftsbezogenen Erwartungen erfüllt.

\section{b) Vertrauen als Antezedenz der Spenderbindung}

Aufgrund der fehlenden Vergleichsmaßstäbe der Leistungen der NPO und ihres immateriellen Charakters basiert die Spender-NPO-Beziehung primär auf Vertrauen. Ohne Vertrauen würde ein Spender eine NPO entweder gar nicht oder nicht auf Dauer unterstützen (Urselmann 1998, S. 235). Durch die systeminhärente Divergenz von Spender und Leistungsempfänger wird Vertrauen als kritische Determinante der Spenderbindung angesehen (Shabbir/Palihawadana/Thwaites 2007, S. 282). Hieraus ergibt sich folgende Hypothese:

H2: Das Vertrauen eines Spenders hat einen positiven Effekt auf die Spenderbindung.

Das Vertrauen eines Spenders beeinflusst jedoch auch indirekt die Spenderbindung. Dies geschieht dadurch, dass Vertrauen das Commitment des Spenders erhöht, welches sich dann - wie gezeigt wurde - positiv auf die Spenderbindung auswirkt. Der innere Bindungszustand des Commitment kann nur entstehen, wenn der Spender der Leistungsfähigkeit und dem Leistungswillen der NPO vertraut (Sargeant/Woodliffe 2007, S. 50). Die Vertrauenswürdigkeit ist somit eine wichtige Voraussetzung für das Entstehen von Commitment (Shabbir/Palihawadana/Thwaites 2007, S. 282). Aus dieser Aussage ergibt sich die dritte Hypothese:

H3: Das Vertrauen eines Spenders hat einen positiven Effekt auf sein Commitment.

\section{Zufriedenheit des Spenders}

\section{a) Explikation des Begriffs Zufriedenheit des Spenders}

Einige Marketingforscher sehen die Kundenzufriedenheit als wichtigste Determinante der Kundenloyalität (Garbarino/Johnson 1999, S. 71; Jones/Sasser 1995, S. 91). Die gestiegene Anzahl an Veröffentlichungen zum Thema Kundenzufriedenheit zeugt zudem von ihrer Bedeutung (Krafft 2007, S. 20; Sauer 2003, S. 230). Charakteristisch ist ein Soll-Ist-Vergleich zwischen einem Vergleichsstandard in Form von Erwartungen und der eigentlichen Leistung (Oliver 1981, S. 25). Zufriedenheit entsteht also immer dann, wenn man erhält, was man erwartet. Damit ergibt sich folgende Definition:

Zufriedenheit ist die affektive Reaktion auf eine in einem komplexen kognitiven Vergleichsprozess zwischen ex-ante Erwartungen und subjektiven Erfahrungen auftretende (Dis-)Konfirmation. 


\section{b) Zufriedenheit als Antezedenz der Spenderbindung}

Im kommerziellen Marketing konnte zwischen Zufriedenheit und Kundenbindung ein enger kausaler Zusammenhang nachgewiesen werden (Britton/Rose 2004, S. 46; Bruhn 1999, S. 194). Zufriedenheit stellt auch eine wichtige Erklärungsgröße für die Spenderbindung dar: Ohne Zufriedenheit sind Aufbau und Erhaltung der Beziehung nicht möglich (Urselmann 1998, S. 73). Positive und befriedigende Erfahrungen mit der NPO veranlassen ihn dazu, die Beziehung beizubehalten (File/Judd/Price 1996, S. 75). Dies führt zur vierten Hypothese:

H4: Die Zufriedenheit eines Spenders hat einen positiven Effekt auf die Spenderbindung.

Die Zufriedenheit eines Spenders beeinflusst jedoch auch indirekt die Spenderbindung. Zufriedenheit stellt neben Vertrauen eine weitere Einflussgröße des Commitment dar (von Stenglin 2008, S. 56). In Untersuchungen des kommerziellen Kundenbindungsmanagement sowie im Nonprofit-Bereich (Swanson/Davis/Zhao 2007, S. $10 \mathrm{ff}$; Bakay/Schwaiger 2006, S. $331 \mathrm{ff}$.) konnte ein direkter positiver Effekt der Zufriedenheit auf das Commitment nachgewiesen werden. Dies führt zur fünften Hypothese:

H5: Die Zufriedenheit eines Spenders hat einen positiven Effekt auf sein Commitment.

Ein weiterer Zusammenhang konnte ebenfalls nachgewiesen werden: Zufriedenheit beeinflusst den Aufbau von Vertrauen (Wünschmann/Müller 2008, S. 148). Vertrauen basiert auf Erfahrungen mit der NPO (Crosby/Evans/Cowles 1990, S. 70). Die über einen Zeitraum gemachten Erfahrungen ermöglichen den Aufbau von Vertrauen; sie tragen dazu bei, dass der Spender entsprechende Erwartungen an die Zuverlässigkeit und die Integrität der NPO entwickelt. Dies führt zur sechsten Hypothese:

H6: Die Zufriedenheit eines Spenders hat einen positiven Effekt auf sein Vertrauen.

\section{Involvement des Spenders}

\section{a) Explikation des Begriffs Involvement des Spenders}

Die vierte untersuchte Einflussgröße der Spenderbindung ist das Involvement, bei welchem die persönliche Relevanz, die wahrgenommene Wichtigkeit bzw. das Interesse gegenüber Sachverhalten (z. B. Produkten) im Vordergrund steht (Mittal 1995, S. 664). Es lassen sich drei Dimensionen des Involvement unterscheiden (Hohl/Naskrent 2009, S. 78): Involvementklasse, -fristigkeit und -art.

Involvement benötigt zunächst einen Auslöser (Jaritz 2008, S. 21). Diese kann man in zwei Klassen einteilen: Transaktion oder Objekt (Hohl/Naskrent 2009, S. 79). Transaktions-Involvement bezeichnet ein vorübergehendes Interesse am Kauf- bzw. Spendenprozess als solchem. Diese Untersuchung schließt das Transaktions-Involvement jedoch aus, da es primär durch Unsicher- 
heit, Zeitmangel oder ähnliche Faktoren entsteht (Michaelidou/Dibb 2008, S. 87). Im Fokus steht vielmehr die unterstützte NPO als sogenanntes Involvement-Objekt.

Als Fristigkeiten lassen sich situatives und dauerhaftes Involvement unterscheiden. Situatives Involvement bezeichnet ein vorübergehendes Interesse; es hat einen temporären Charakter und nimmt i. d. R. schnell wieder ab (Homburg/Kebbel 2001, S. 45). Dauerhaftes Involvement existiert hingegen unabhängig von bestimmten Situationen, da es durch eine zentrale Bedeutung für den Konsumenten entsteht. Je stärker das Bezugsobjekt die zentralen Eigenschaften des Konsumenten berührt, desto höher ist das dauerhafte Involvement (Mittal/Lee 1989, S. 365). Da es damit das Interesse bezeichnet, ein finanzieller Unterstützer der NPO zu sein, wird es im Folgenden näher untersucht und das situative Involvement bewusst außen vor bleiben.

Die dritte Dimension ist die Involvementart mit drei Ausprägungsformen (Hohl/Naskrent 2009, S. 83 f.). Zunächst kann Involvement kognitiv sein. Involvierte Spender sind bei der Informationssuche, -verarbeitung und -speicherung stark engagiert. Je höher das Involvement ist, desto intensiver fällt die Suche, Verarbeitung und Speicherung von Informationen aus (Gordon/ McKeage/Fox 1998, S. 445). Es trägt aber nicht zur Erklärung der Spenderbindung bei. Vielmehr gilt es, das emotionale Involvement zu berücksichtigen, welches mit den persönlichen Werten und Einstellungen des Spenders in Verbindung steht und ein hohes Engagement im Sinne einer empfundenen persönlichen Relevanz bedeutet (Esch/Hardiman/Kiss 2002, S. 237). Die dritte Involvementart, das konative Involvement, zeichnet sich durch eine Bereitschaft zu außergewöhnlichem Aufwand für das Bezugsobjekt aus. Es findet hier keine Berücksichtigung, da keine Trennschärfe zum beabsichtigten Spenderverhalten besteht.

Aufbauend auf den beschriebenen Merkmalen wird Involvement wie folgt definiert:

Spenderinvolvement ist das dauerhafte Interesse des Spenders an der NPO und die von ihm wahrgenommene persönliche Relevanz der NPO, welche sich in einer emotionalen Aktivierung und Motivation des Spenders äußert.

\section{b) Involvement als Antezedenz der Spenderbindung}

Generell erscheint es sinnvoll, Involvement als Antezedenz für die Kundenbindung zu sehen. Insbesondere das dauerhafte Involvement weist eine positive Bedeutung für die Loyalität auf (Homburg/Kebbel 2001, S. 49). Sieht man eine positive Einstellung als Voraussetzung für die Bindung an, so lässt sich Letztere nur bei einem dauerhaften und emotionalen Involvement finden (Dick/Basu 1994, S. 99 ff.). Ohne Involvement des Spenders wären keine Spendeneinnahmen zu verzeichnen. Dieser Zusammenhang führt zu folgenden Hypothesen:

H7: Das Spenderinvolvement hat einen positiven Effekt auf die Spenderbindung.

Das Spenderinvolvement wirkt sich auch indirekt auf die Spenderbindung aus. Hier ist zunächst der positive Einfluss des dauerhaften Spenderinvolvement auf das Commitment des Spenders herauszustellen (Gordon/McKeage/Fox 1998, S. 447). Das Involvement ist zentrale Voraussetzung für die innere Verbundenheit des Spenders (Ahearne/Bhattacharya/Gruen 2005, S. 575). Jeker (2002, S. 117) formuliert den Sachverhalt wie folgt: „Commitment existiert nur, wenn die 
Beziehung als wichtig erachtet wird." Das Involvement hat somit Einfluss auf die Bereitschaft, eine Bindung einzugehen, was zur 8. Hypothese führt.

H8: Das Spenderinvolvement hat einen positiven Effekt auf das Commitment.

Das Spenderinvolvement beeinflusst darüber hinaus die Zufriedenheit. Es wirkt auf alle Komponenten des Modells der Entstehung der Zufriedenheit ein. Selbst die Stärke der empfundenen Diskrepanz der Erwartungen und der wahrgenommenen Leistung hängt vom Involvement ab (Jeker 2002, S. 132). Das Involvement stellt folglich eine deterministische Größe der Zufriedenheit dar und mediiert somit Commitment. Weiterhin macht sich der Spender z. B. durch die intensive Auseinandersetzung mit der Spende bewusst, welche wertvolle Unterstützung er leistet. Dies fördert seine Zufriedenheit bzw. lässt sie durch hohes Involvement länger anhalten. Weiterhin wird der Zusammenhang damit begründet, dass sich der Spender bei zunehmendem Involvement auf ein Zufriedenheitsniveau festlegt bzw. nur noch Zufriedenheitssteigerungen wahrnimmt, selbst wenn die wahrgenommene Leistung kurzfristig nachlässt (Bennett/Ali-Choudhury 2009, S. 164). Die Abhängigkeit beschreibt folgende Hypothese:

H9: Das Spenderinvolvement hat einen positiven Effekt auf seine Zufriedenheit.

Nachdem die vermuteten Antezedenzbeziehungen für die in diesem Artikel untersuchten verhaltenswissenschaftlichen Determinanten der Spenderbindung jeweils einzeln theoretisch hergeleitet wurden, gilt es, diese empirisch zu überprüfen.

\section{Empirische Analyse der Spenderbindung}

\section{Konzeption der Befragung und Untersuchungsvorgehen}

Die empirische Analyse zielt darauf ab, Probanden, die NPOs finanziell in Form einer Spende unterstützen, über ihr Verhalten zu befragen. Die Befragung konzentriert sich somit auf Personen, die für eine NPO spenden. Im Gegensatz zur Konsumgüterbranche mit einer großzahligen Grundgesamtheit ist die Zahl der möglichen Testpersonen aus dem Spendenmarkt entsprechend begrenzt. Darüber hinaus gilt, dass insbesondere ältere Menschen spenden. Die Besonderheiten begründen auch trotz des hohen Aufwands die Entscheidung für eine Telefonbefragung.

Die Auswahl der Probanden erfolgte in Kooperation mit verschiedenen NPOs. Das Ziel der Zusammenarbeit stellte der Zugang zu Spenderdaten dar, mit denen eine zielgenaue Akquise der Probanden ohne große Streuverluste erfolgen konnte. Insgesamt wurden vier NPOs gewonnen. Es handelt sich hierbei um soziale NPOs, die wohltätiges und gemeinnütziges Engagement für Menschen und Natur leisten.

Zur Sicherstellung der Qualität der Befragung wurden zwei verschiedene Pretests durchgeführt. Bei dem inhaltlichen Pretest bewerteten Personen mit fundiertem Marketing-Wissen die Zusammenhänge der vier Einflussgrößen und den gewählten Fragestellungen, d. h. ob Definition und Wortwahl übereinstimmen. Der formale Pretest verfolgte das Ziel, die Nachvollziehbarkeit des 
Aufbaus der Befragung und die Verständlichkeit der Anweisungen sicherzustellen. Insgesamt führten die Ergebnisse der Pretests nur zu kleineren notwendigen Veränderungen der Formulierungen der Items. Der finale Wortlaut der Fragen, deren Formulierung in Anlehnung an bereits erfolgreich durchgeführten Befragungen angelehnt wurde, befindet sich im Anhang.

Insgesamt wurden 1028 Spender per Zufallsauswahl von den durch die kooperierenden NPOs zur Verfügung gestellten Kontaktdaten angerufen. 439 Probanden erklärten sich bereit, an der Befragung teilzunehmen, 364 Probanden führten sie bis zum Ende durch. Das Durchschnittsalter der Stichprobe liegt bei 64 Jahren und die Geschlechterverteilung ist mit 51 \% weiblichen Probanden ausgewogen. Die Stichprobe beinhaltet auch ehemalige Spender, um verschiedene, d. h. abweichende Antwortmuster bei der Befragung hervorzurufen. Als ehemaliger Spender gilt, wer 18 Monate nicht an die spezifische NPO gespendet hat (Bennett/Ali-Choudhury 2009, S. 170). Insgesamt haben 47 ehemalige Spender (dies entspricht $13 \%$ aller vollständigen Interviews) an der Befragung teilgenommen. Eine Analyse der fehlenden Werte lieferte keine Anhaltspunkte für ein systematisches Auftreten.

Um Wirkungsbeziehungen und Interdependenzen simultan zu testen, wendet die Marketingwissenschaft i. d. R. ein Kausalmodell an (Giere/Wirtz/Schilke 2006, S. 683). Kausalmodelle wurden früher überwiegend mittels kovarianzbasierten Ansätzen untersucht, die durch die fortgeschrittene Softwareentwicklung breite Anwendung finden. Einen alternativen Ansatz für die Berechnung von Kausalmodellen stellt der varianzbasierte Partial Least Squares (PLS-)Ansatz dar, der insbesondere aufgrund der mittleren Stichprobengröße, des prognostizierenden Ansatzes und der unterschiedlichen Dimensionen, aus denen sich die Konstrukte Commitment und Vertrauen konstituieren, zur Analyse mittels SmartPLS 2.0 (Ringle/Wende/Will 2005) gewählt wurde (Jaritz 2008, S. 160; Jahn 2007, S. 3).

\section{Befunde}

Der erste Schritt einer Analyse empirischer Daten besteht in der Gütebeurteilung, d. h. der Reliabilität und Validität, der gemessenen Konstrukte. Unter Validität versteht man den Grad, in dem die Items die Bedeutung des Konstrukts abbilden (Mittal 1995, S. 665). Diese Zugehörigkeitsstärke wird mithilfe der Faktorenanalyse gemessen. Die jeweiligen Items werden dabei auf Eindimensionalität geprüft. Hierbei gilt es zu untersuchen, ob sie am höchsten auf die ursprünglich zugeordneten Konstrukte laden (Mittal 1995, S. 665). In der vorgenommenen Evaluierung zeigen alle abgefragten Konstrukte eine eindimensionale Faktorstruktur auf. Die entsprechenden Items laden am höchsten auf die ursprünglich zugeordneten Konstrukte und erreichen keinen höheren Wert für ein anderes Konstrukt. Insgesamt weisen alle Faktorladungen hohe Werte auf.

Zusätzlich zur Faktorenanalyse, welche auf Ebene der einzelnen Items stattfindet, wurde die Güte durch Analysen auf Ebene der Messmodelle ermittelt, d. h. in wieweit sämtliche Items das Konstrukt repräsentieren. Die interne Konsistenz stellt ein Gütemaß für die Homogenität einer Skala dar. Eine hohe interne Konsistenz bedeutet, dass die Items im Wesentlichen das Gleiche messen.

Die interne Konsistenz kann Werte zwischen 0 und 1 annehmen, wobei ein höherer Wert eine höhere Güte darstellt. 0,6 stellt den allgemein anerkannten Grenzwert dar (Bagozzi/Yi 1988, 
S. 82). Die vorliegende Untersuchung liefert Ergebnisse von mindestens 0,8, was Abb. 2 verdeutlicht.

Neben der internen Konsistenz stellt Cronbach's Alpha ein weitverbreitetes Gütemaß für die Konstruktreliabilität dar. Cronbach's Alpha repräsentiert den Anteil der Gesamtvarianz, der sich auf einen gemeinsamen Faktor zurückführen lässt. Es kann Werte zwischen 0 und 1 annehmen, wobei hohe Werte für hohe Korrelationen zwischen den Items sprechen. Hinsichtlich der als akzeptabel anzusehenden Mindestwerte gibt die Literatur einen Wert von 0,7 vor (Thomas/Cunningham/Williams 2002). In der vorliegenden Untersuchung haben sowohl alle Determinanten der Spenderbindung als auch das Zielkonstrukt an sich (Verhaltensabsicht) eine hohe Reliabilität im Sinne des Cronbach's Alpha (siehe Abb. 2).

Neben der internen Konsistenz und Cronbach's Alpha stellt die durchschnittlich erfasste Varianz (DEV) ein weiteres Beurteilungskriterium dar. Hiermit lässt sich überprüfen, wie hoch der durch ein Item erklärte Varianzanteil einer exogenen latenten Variablen ausfällt. Konkret misst die DEV die Varianz, die durch die Indikatoren relativ zum Messfehler erklärt wird. Die DEV soll mindestens einen Wert von 0,5 annehmen (Bagozzi/Yi 1988, S. 82; Fornell/Larcker 1981, S. 46). Abb. 2 zeigt, dass alle Determinanten der Spenderbindung über eine hohe Konstruktreliabilität im Sinne der DEV verfügen. Allerdings liegt der Wert für das zu erklärende Konstrukt Verhaltensabsicht des Spenders geringfügig unter der geforderten Mindestgröße. Da es jedoch alle bisherigen Validitäts- und Reliabilitätsanforderungen sehr gut erfüllt, gilt dies als unproblematisch. Andere Forscher sehen in Ausnahmefällen Werte zwischen 0,4 und 0,5 als ausreichend an (Homburg/ Giering 2001, S. 17).

\begin{tabular}{|l|c|c|c|c|}
\hline & Anzahl Items & $\begin{array}{c}\text { Interne } \\
\text { Konsistenz } \\
(>0,6)\end{array}$ & $\begin{array}{c}\text { Cronbach's } \\
\text { Alpha } \\
(>0,7)\end{array}$ & $\begin{array}{c}\text { DEV } \\
(>0,5)\end{array}$ \\
\hline ABSICHT & 6 & 0,80 & 0,71 & 0,41 \\
\hline NORMATIV & 5 & 0,86 & 0,79 & 0,54 \\
\hline KALKULATIV & 5 & 0,88 & 0,83 & 0,59 \\
\hline AFFEKTIV & 5 & 0,85 & 0,78 & 0,53 \\
\hline ZUTRAUEN & 5 & 0,91 & 0,88 & 0,68 \\
\hline VERLASS & 5 & 0,90 & 0,87 & 0,65 \\
\hline ZUFRIEDEN & 4 & 0,90 & 0,85 & 0,70 \\
\hline INVOLV & 4 & 0,87 & 0,79 & 0,62 \\
\hline
\end{tabular}

Abb. 2: Beurteilungsergebnisse der Messmodelle

Quelle: Eigene Darstellung

Der nächste Schritt im Rahmen der Gütebeurteilung beinhaltet die Ermittlung der Diskriminanzvalidität. Diskriminanzvalidität besteht, wenn die latente Variable die Varianz ihrer eigenen Items besser erklärt als die Varianz einer anderen latenten Variablen. Die Diskriminanzvalidität baut auf der DEV auf. Die Wurzel der DEV eines Konstrukts sollte im Rahmen der Diskriminanzva- 
lidität höher sein als die Korrelationen zwischen dem Konstrukt und anderen Konstrukten im Modell (Fornell/Larcker 1981, S. 46). Zur Veranschaulichung dient eine Korrelationsmatrix (Abb. 3). Die Matrix zeigt zunächst, dass alle Fälle die empfohlene maximale Korrelation zwischen unterschiedlichen Konstrukten von 0,9 unterschreiten (Hulland 1999, S. 200). Darüber hinaus verdeutlicht die Matrix, dass die Wurzel der DEV der Konstrukte in den meisten Fällen größere Werte aufweist als die Korrelationen zwischen den anderen latenten Variablen. Lediglich zwischen Involvement und affektivem Commitment sowie zwischen Zutrauen und Verlass sind die Korrelationen um 0,02 bzw. 0,06 zu groß. Solche Werte gelten in anderen empirischen Studien jedoch als unkritisch (Lüthje 2008, S. 138). Damit lässt sich die Diskriminanzvalidität als ausreichend bezeichnen.

\begin{tabular}{|l|c|c|c|c|c|c|c|c|}
\hline & ABSICHT & AFFEKTIV & INVOLV & KALKULATIV & NORMATIV & VERLASS & ZUFRIEDEN & ZUTRAUEN \\
\hline $\begin{array}{c}\text { Wurzel von } \\
\text { DEV }\end{array}$ & $\mathbf{0 , 6 4}$ & $\mathbf{0 , 7 3}$ & $\mathbf{0 , 7 9}$ & $\mathbf{0 , 7 7}$ & $\mathbf{0 , 7 4}$ & $\mathbf{0 , 8 1}$ & $\mathbf{0 , 8 4}$ & $\mathbf{0 , 8 3}$ \\
\hline ABSICHT & 1 & 0 & 0 & 0 & 0 & 0 & 0 & 0 \\
\hline AFFEKTIV & 0,52 & 1 & 0 & 0 & 0 & 0 & 0 & 0 \\
\hline INVOLV & 0,43 & 0,75 & 1 & 0 & 0 & 0 & 0 & 0 \\
\hline KALKULATIV & 0,30 & 0,56 & 0,50 & 1 & 0 & 0 & 0 & 0 \\
\hline NORMATIV & 0,43 & 0,71 & 0,66 & 0,46 & 1 & 0 & 0 & 0 \\
\hline VERLASS & 0,41 & 0,40 & 0,40 & 0,20 & 0,44 & 1 & 0 & 0 \\
\hline ZUFRIEDEN & 0,45 & 0,51 & 0,50 & 0,31 & 0,53 & 0,80 & 1 & 0 \\
\hline ZUTRAUEN & 0,41 & 0,41 & 0,38 & 0,17 & 0,46 & 0,87 & 0,81 & 1 \\
\hline
\end{tabular}

Abb. 3: Diskriminanzvalidität

Quelle: Eigene Darstellung

Nachdem auf diese Art und Weise bei jedem einzelnen Konstrukt eine zufriedenstellende Messgüte ermittelt wurde, gilt es nun, die Mehrdimensionalität der Konstrukte Commitment und Vertrauen zu betrachten. Dies erfolgt durch die Ermittlung der Faktorladungen und Signifikanzen der einzelnen Dimensionen. Abb. 4 zeigt, dass alle drei Dimensionen des Konstrukts Commitment und beide Dimensionen des Konstrukts Vertrauen jeweils vergleichbare Ladungen aufweisen. Sie bestimmen damit zu gleichen Teilen das Commitment bzw. Vertrauen.

\begin{tabular}{|l|c|c|}
\hline Beziehung & Faktorladungen & Signifikanz \\
\hline Normativ $\rightarrow$ Commitment & 0,3922 & ${ }^{* * *}$ \\
\hline Kalkulativ $\rightarrow$ Commitment & 0,3745 & ${ }^{* * *}$ \\
\hline Affektiv $\rightarrow$ Commitment & 0,4088 & ${ }^{* * *}$ \\
\hline Zutrauen $\rightarrow$ Vertrauen & 0,5272 & ${ }^{* * *}$ \\
\hline Verlass $\rightarrow$ Vertrauen & 0,5058 & ${ }^{* * *}$ \\
\hline
\end{tabular}

Abb 4: Analyseergebnisse der mehrdimensionalen Konstrukte

Quelle: Eigene Darstellung 
Als letzter Analyseschritt wurden Stärke und Signifikanz der Wirkungsbeziehungen analysiert und damit die postulierten Hypothesen schließlich näher betrachtet. Die gemessene Stärke der Pfadkoeffizienten, die im Spektrum -1 bis +1 liegen kann, lässt sich wie folgt interpretieren: Negative Werte bedeuten eine zu den Hypothesen konträre Wirkungsbeziehung (Jahn 2007, S. 10). Werte nahe Null bringen einen schwachen Erklärungsbeitrag einer latenten Variablen zum Ausdruck, während ein Wert nahe Eins einen starken Zusammenhang impliziert. Allerdings sind bereits Werte größer als 0,4 in komplexeren Modellen als hoch zu beurteilen. Besonders im Grenzbereich $(0,15-0,2)$ erscheint eine zusätzliche Aussage, ob eine Wirkungsbeziehung „echt“ ist, mithilfe der Signifikanz hilfreich (Jahn 2007, S. 10, 18). Pfade, die keine Signifikanz aufweisen oder ein anderes Vorzeichen haben, widerlegen die Hypothesen. Abb. 5 zeigt die Ergebnisse und führt weiterhin (innerhalb der Ovale), die Werte der jeweiligen Bestimmtheitsmaße auf. Das Bestimmtheitsmaß bzw. $\mathrm{R}^{2}$ stellt hierbei den Anteil der erklärten Varianz des latenten Konstrukts dar. Dies bedeutet, dass das $\mathrm{R}^{2}$ Auskunft gibt über die Wirkungsstärke aller exogenen Konstrukte, die ein endogenes Konstrukt erklären (Jaritz 2008, S. 172). Der mögliche Wertebereich des $\mathrm{R}^{2}$ liegt zwischen 0 und 1. Je größer der Wert, desto größer ist die erklärte Streuung. Werte ab 0,3 gelten als durchschnittlich und Werte unter 0,19 als schwach. Dem Modell lässt sich dann eine nennenswerte Erklärungskraft attestieren, wenn, wie bei der vorliegenden Untersuchung der Fall, das Zielkonstrukt (hier Spenderbindung) ein $\mathrm{R}^{2}$ von mindestens 0,3 aufweist.

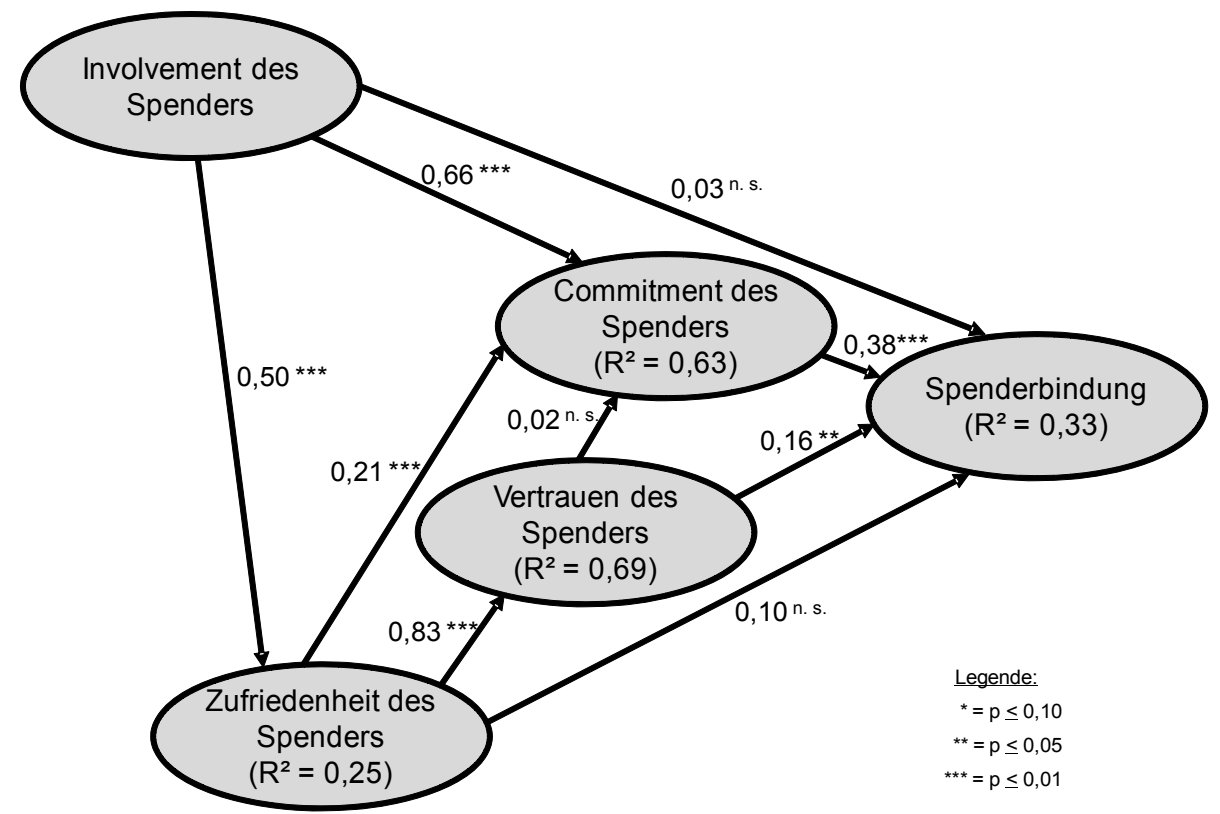

Abb. 5: Ergebnisse des Gesamtmodells

Quelle: Eigene Darstellung 


\section{Interpretation}

Insgesamt halten sechs von neun Hypothesen der empirischen Prüfung stand, was eine nomologische Validität des Kausalmodells bedeutet. Auch wenn bestimmte Determinanten (Involvement und Zufriedenheit) keinen direkten Einfluss auf die Spenderbindung haben, wirken diese indirekt auf die Spenderbindung.

Involvement stellt laut den Ergebnissen der Untersuchung keine direkte Determinante der Spenderbindung dar (ursprünglich $\mathrm{H}_{7}$ ). Eine theoretische Erklärung für diesen Sachverhalt könnte darin liegen, dass NPOs und das Thema Spenden extremere Ausprägungen des Involvement hervorrufen als Konsumsituationen, bei denen Ausprägungen im Mittelbereich vorliegen (Rothschild 1979, S. 13). Involvement hat hier eine eher bipolare Ausprägung: Entweder zeigen Menschen ein extrem hohes Involvement für die Organisation und sind dann bereit, sich emotional dafür zu engagieren und finanzielle Unterstützung zu leisten oder sie zeigen überhaupt kein Involvement für die Organisation und ihren Tätigkeitsbereich, sodass sie es nie in Betracht ziehen würden zu spenden (Rothschild 1979, S. 13 f.). Involvement stellt somit eine Grundvoraussetzung dar, die für die Spenderbindung keine direkte, sondern nur eine indirekte Bedeutung hat. Da die Untersuchung sich größtenteils auf aktive Spender fokussierte, kann man davon ausgehen, dass diese ein sehr hohes Involvement für die NPO aufweisen.

Die Tatsache, dass die Spenderzufriedenheit keinen großen Einfluss auf die Spenderbindung hat (ursprünglich $\mathrm{H}_{4}$ ), zeigt, dass das Konstrukt nicht die Bedeutung besitzt, welche die Forschung ihm generell zuschreibt. Auch Untersuchungen im kommerziellen Marketing kommen zu dem Ergebnis, dass die Kundenzufriedenheit nicht den Stellenwert für die Kundenbindung einnimmt, wie es häufig postuliert wird (Herrmann/Huber/Braunstein 2000, S. 294). Ebenso wurde dies bereits für die Spender-NPO-Beziehung vermutet (Sargeant 2008, S. 4). Zwei empirische Untersuchungen bestätigten diesen Sachverhalt durch einen insignifikanten Einfluss der Spenderzufriedenheit auf das Spenderverhalten (Michalski/Helmig 2010, S. 242; Arnett/German/Hunt 2003, S. 97 ff.). Abschließend hielten die Autoren (Arnett/German/Hunt 2003, S. 100) jedoch fest, dass das Ergebnis ihrer Untersuchung nicht die generelle Bedeutung der Zufriedenheit für die Austauschbeziehung zwischen Spender und NPO negiere. Auch wenn die Spenderzufriedenheit in ihrer Untersuchung keine Auswirkung auf das Zielkonstrukt hatte, so vermuten sie, dass die Spenderzufriedenheit als indirekte Determinante, z. B. über das Commitment, eine Bedeutung hat. Dies bestätigt die vorliegende Analyse.

\section{Managementimplikationen und Fazit}

Da alle vier Determinanten laut der Untersuchung eine Daseinsberechtigung haben, sollen im Folgenden Maßnahmen aufgezeigt werden, mit denen diese gleichzeitig gefördert werden können. Viele Maßnahmen, die NPOs heutzutage anwenden, eignen sich nur zur Verstärkung einzelner Einflussgrößen, wie z. B. Rechenschaftsberichte (Stärkung des Vertrauens) oder Mitleid erregende Botschaften (Erhöhung des normativen Commitment und Involvement) (Haibach 2006, 
S. 46). Die Maßnahmen Erfolgsmeldungen und Dank ermöglichen es hingegen alle vier Determinanten gleichzeitig zu stärken.

Spender möchten am Nutzenzuwachs der Hilfsbedürftigen partizipieren, da „Menschen für Menschen spenden“ (Hohn 2001, S. 50). Spender erhalten eine immaterielle Gratifikation, wenn sie erfahren, wie die Spende dem Empfänger tatsächlich geholfen hat. Sie wollen erfahren, ob und wie die NPO ihr (gemeinsames) Ziel erreicht hat (Sargeant 2008, S. 15). Die NPO sollte deswegen ihren Spendern regelmäßig aufzeigen, welchen Unterschied ihre Unterstützung bewirkt, welche Leistungen sie damit erbringt und wie der Einzelne durch seine Spende konkrete Hilfestellung ermöglicht (Bennett/Ali-Choudhury 2009, S. 167). Im Rahmen einer auf Spenderbindung abzielenden Fundraising-Praxis ist es unabdingbar, dem Spender mit entsprechenden qualitativen, emotionalen Erfolgsmeldungen aufzuzeigen, welchen Mehrwert die Arbeit der NPO erbracht hat, und ihn damit zu überzeugen, weiter zu spenden. Berichte über bisherige Erfolge und Tätigkeiten der NPO wirken dissonanzreduzierend und somit zufriedenheitserhöhend. Darüber hinaus kann die NPO mit Erfolgsmeldungen ihre Kompetenz sowie ihre Glaubwürdigkeit und Verlässlichkeit unter Beweis stellen und damit das Vertrauen der Spender stärken. Durch Informationen über die Auswirkungen ihrer Spenden lässt eine NPO die Förderer zudem an den Erfolgen ihrer Projekte teilhaben und bringt zum Ausdruck, dass diese Erfolge ohne die Unterstützung der Spender nicht möglich wären. Dies schafft ein Identifikationspotenzial und Zugehörigkeitsgefühl, was das affektive Commitment erhöht. Durch entsprechende positive Erfolgsmeldungen lässt sich zum anderen das Gefühl der Spender verstärken, dass die Beendigung ihres Engagements für die Leistungsempfänger einen Verlust bedeuten würde. Somit fördern Erfolgsmeldungen auch das normative Commitment. Letztendlich lässt sich auch das Involvement der Spender durch Erfolgsmeldungen vergrößern. Je mehr die Spender über die Organisation und ihre Projekte erfahren, umso mehr interessieren sie sich dafür und beschäftigen sich damit (Hohn 2001, S. 61).

Als ein weiteres wichtiges Prinzip im Rahmen des Spenderbindungsmanagement gilt die Wertschätzung der geleisteten Unterstützung. Jeder Spender wünscht sich bewusst oder unbewusst Anerkennung für sein gemeinnütziges Engagement (Andreasen/Kotler 2008, S. 371). Viele Organisationen schaffen es jedoch offenbar nicht, das Bedürfnis ihrer Spender nach Dank und Anerkennung zu erfüllen (Urselmann 2007, S. 33). Nicht selten erhalten Spender nach einer Spende überhaupt keine Reaktion von der NPO (Andreasen/Kotler 2008, S. 372). Durch einen glaubwürdigen Dank lässt sich zunächst die Zufriedenheit des Spenders fördern (Haibach 2006, S. 356). Die Unsicherheit nach der Spende lässt sich von der NPO durch den Dank abbauen. Ähnlich wie bei Erfolgsmeldungen bestätigt die NPO den Spendern, die richtige Entscheidung getroffen zu haben (Urselmann 2007, S. 67 f.). Eine Danksagung stiftet dem Spender darüber hinaus sowohl einen immateriellen als auch einen materiellen Nutzen und fördert somit sein kalkulatives Commitment. Zudem vermittelt dies Verbundenheit im Sinne des affektiven Commitment, da sie dem Spender signalisiert, dass die NPO ihn als Partner und nicht als Geldquelle sieht. Die persönliche Relevanz fördert zudem das Involvement. Ein zeitnah ausgesprochener Dank vermittelt dem Spender letztendlich das Gefühl, dass die NPO verlässlich und kompetent handelt und erhöht damit das Spendervertrauen.

Die Ausführungen über die Wirkungen der Maßnahmen Dank und Erfolgsmeldungen, unterstützen NPOs, ein erfolgreiches Spenderbindungsmanagement zu etablieren. Damit zeigt dieser Beitrag konkrete Marketingmaßnahmen und Problemlösungshilfen für die Praxis auf, ohne die theo- 
retische Perspektive in der Argumentation zu vernachlässigen. Der Artikel bietet somit ein umfassendes Verständnis für Spenderbindung.

Allerdings stellt die Übertragbarkeit der Ergebnisse wie bei vielen Studien eine Herausforderung dar (Ahearne/Bhattacharya/Gruen 2005, S. 581). Die Daten wurden ausschließlich in Deutschland erhoben. Es ist aber durchaus möglich, dass in anderen Ländern die Bedeutung der Determinanten variiert (Sargeant/Woodliffe 2007, S. 63). Die Untersuchung stellt außerdem eine einmalige (Querschnitts-)Untersuchung dar, die lediglich eine zeitpunktbezogene, statische Betrachtung zulässt; Aussagen über die Zeitkonsistenz der ermittelten Einflussgrößen sind somit nicht möglich. Diese Grenzen der Arbeit bieten Anknüpfungspunkte für weitere Forschungsaktivitäten. Beispielsweise wäre eine Bestätigung des Modells im Rahmen einer Replikationsstudie (im Ausland) ein wertvoller Erkenntnisgewinn. Eine weitere Forschungsaufgabe besteht darin, die Spenderbindung in einer Langzeitstudie zu untersuchen.

Solche Forschungsaktivitäten stellen vor dem Hintergrund des zunehmenden Drucks auf NPOs einen wertvollen Beitrag dar. Einerseits müssen NPOs auch in Zukunft mit weiter ansteigender Wettbewerbsintensität rechnen; andererseits erfordern die zunehmenden gesellschaftspolitischen Probleme (z. B. Erderwärmung) in Zukunft ein verstärktes Engagement von NPOs. Die Arbeit von NPOs wird in den nächsten Jahrzehnten also weiterhin an Bedeutung gewinnen. Im Rahmen dessen lässt sich davon ausgehen, dass die Spenderbindung - auf welche die in dieser Arbeit untersuchten Determinanten einwirken - und die entsprechenden Maßnahmen in den kommenden Jahren aufgrund ihrer zunehmenden Notwendigkeit sowohl in der Forschung als auch in der Praxis eine größere Verbreitung finden. 


\section{Anhang: Wortlaut der Fragestellungen}

\begin{tabular}{|c|c|c|c|}
\hline Einflussgröße & $\begin{array}{l}\text { Facette/ } \\
\text { Dimension }\end{array}$ & Wortlaut der Items & $\begin{array}{l}\text { Quelle } \\
\text { (in Anlehnung an) }\end{array}$ \\
\hline \multirow{3}{*}{$\begin{array}{l}\text { Commitment des } \\
\text { Spenders }\end{array}$} & $\begin{array}{l}\text { Normatives } \\
\text { Commitment }\end{array}$ & $\begin{array}{l}\text { Ich empfinde ein Gefühl der Verantwortung gegenüber der } \\
\text { NPO. } \\
\text { Meiner Meinung nach erfordert es der Anstand, sich als } \\
\text { Spender gegenüber der NPO loyal zu verhalten. } \\
\text { Bei der Beendigung meiner finanziellen Unterstützung für } \\
\text { die NPO hätte ich Gewissensbisse. } \\
\text { Ich fühle mich dazu verpflichtet, die NPO finanziell zu un- } \\
\text { terstützen. } \\
\text { Der Abbruch der Beziehung zur NPO wäre nicht fair, weil } \\
\text { die NPO meine Treue verdient hat. }\end{array}$ & $\begin{array}{l}\text { Lomberg (2008); } \\
\text { Allen/Meyer (1990) }\end{array}$ \\
\hline & $\begin{array}{l}\text { Kalkulatives } \\
\text { Commitment }\end{array}$ & $\begin{array}{l}\text { Die Beendigung meiner finanziellen Unterstützung für die } \\
\text { NPO wäre ein persönlicher Verlust, d. h. eine Verschlech- } \\
\text { terung für mich. } \\
\text { Die Beziehung zur NPO ist für mich vorteilhaft. } \\
\text { Durch meine Spende an die NPO profitiere ich auch selbst. } \\
\text { Ich verdanke meiner Beziehung zur NPO viele Vorzüge. } \\
\text { Das Einstellen meiner Spenden an die NPO wäre für mich } \\
\text { mit Nachteilen verbunden. }\end{array}$ & $\begin{array}{l}\text { Sargeant/Lee (2002 a); Green/ } \\
\text { Webb (1997); } \\
\text { Allen/Meyer (1990) }\end{array}$ \\
\hline & $\begin{array}{l}\text { Affektives } \\
\text { Commitment }\end{array}$ & $\begin{array}{l}\text { Ich bin stolz darauf, ein Spender der NPO zu sein. } \\
\text { Ich fühle mich mit der NPO verbunden. } \\
\text { Ich empfinde ein gewisses Zugehörigkeitsgefühl zur NPO. } \\
\text { Ich identifiziere mich mit der NPO. } \\
\text { Bei Kritik an der NPO oder bei Problemen fühle ich mich } \\
\text { persönlich betroffen. }\end{array}$ & $\begin{array}{l}\text { Süß (2007); } \\
\text { Johnson/Garbarino (2001); } \\
\text { Allen/Meyer (1990) }\end{array}$ \\
\hline \multirow[t]{2}{*}{$\begin{array}{l}\text { Vertrauen des } \\
\text { Spenders }\end{array}$} & Zutrauen & $\begin{array}{l}\text { Meiner Meinung nach ist die NPO kompetent. } \\
\text { Ich habe das Gefühl, dass die NPO ihr Handwerk versteht. } \\
\text { Ich traue der NPO zu, die Ziele zu erreichen, die sie sich } \\
\text { setzt. } \\
\text { Ich bin überzeugt davon, dass die NPO imstande ist, ihre } \\
\text { Versprechen zu halten. } \\
\text { Meiner Meinung nach besitzt die NPO die Fähigkeiten und } \\
\text { die Eignung, zuverlässig zu handeln. }\end{array}$ & $\begin{array}{l}\text { Sargeant/Lee (2002 a); } \\
\text { Graeff (1998) }\end{array}$ \\
\hline & Verlass & $\begin{array}{l}\text { Meiner Meinung nach ist die NPO vertrauenswürdig. } \\
\text { Ich denke, dass die NPO ehrlich zu ihren Spendern ist. } \\
\text { Ich kann mich auf die NPO verlassen. } \\
\text { Ich bin überzeugt davon, dass die NPO gewillt ist, ihre } \\
\text { Versprechen zu halten. } \\
\text { Die NPO handelt uneigennützig. }\end{array}$ & $\begin{array}{l}\text { Sargeant/Woodliffe (2007); } \\
\text { Wilke (2007); } \\
\text { Doney/Cannon (1997) }\end{array}$ \\
\hline $\begin{array}{l}\text { Zufriedenheit des } \\
\text { Spenders }\end{array}$ & - & $\begin{array}{l}\text { Die NPO leistet all das, was ich von dieser Organisation } \\
\text { erhoffe. } \\
\text { Die NPO erfüllt meine Erwartungen voll und ganz. } \\
\text { Die Leistung der NPO entspricht meinen Idealvorstellun- } \\
\text { gen. } \\
\text { Insgesamt bin ich sehr zufrieden mit der NPO. }\end{array}$ & $\begin{array}{l}\text { Jaritz (2008); } \\
\text { Markert (2008); } \\
\text { Jeker (2002) }\end{array}$ \\
\hline $\begin{array}{l}\text { Involvement des } \\
\text { Spenders }\end{array}$ & $\begin{array}{l}\text { Emotionales, dauer- } \\
\text { haftes Involvement }\end{array}$ & $\begin{array}{l}\text { Die NPO hat für mich einen großen Stellenwert. } \\
\text { Die NPO ist für mich wichtig und bedeutet mir viel. } \\
\text { Die NPO spielt eine große Rolle in meinem Leben. } \\
\text { Ich interessiere mich sehr für die NPO. }\end{array}$ & $\begin{array}{l}\text { Jaritz (2008); } \\
\text { Thomas/Cunningham/Wil- } \\
\text { liams (2002); } \\
\text { Mittal/Lee (1989) }\end{array}$ \\
\hline Zielvariable & $\begin{array}{l}\text { Facette/ } \\
\text { Dimension }\end{array}$ & Wortlaut der Items & \\
\hline $\begin{array}{l}\text { Spenderbindung } \\
\text { (Verhaltensab- } \\
\text { sicht) }\end{array}$ & - & $\begin{array}{l}\text { Beabsichtigen Sie, in Zukunft diese NPO weiterhin finan- } \\
\text { ziell zu unterstützen? } \\
\text { Wollen Sie langfristig ein Spender von dieser NPO blei- } \\
\text { ben? } \\
\text { Könnten Sie sich vorstellen, in Zukunft mehr für die NPO } \\
\text { zu spenden? } \\
\text { Ziehen Sie in Erwägung, neben den bisherigen auch andere } \\
\text { Projekte der NPO demnächst finanziell zu unterstützen? } \\
\text { Beabsichtigen Sie, Ihre Freunde und Bekannte in Zukunft } \\
\text { zu ermutigen, die NPO ebenfalls finanziell zu unterstützen? } \\
\text { Haben Sie vor, Ihre positiven Erfahrungen mit der NPO an } \\
\text { andere weiterzugeben? }\end{array}$ & $\begin{array}{l}\text { Neumann (2007); } \\
\text { Sargeant/Lee (2004 a); } \\
\text { Teichert/Rost (2003) }\end{array}$ \\
\hline
\end{tabular}




\section{Donor retention - behavioral determinants and implications for Nonprofit-Organizations}

behavioral determinants; donor loyalty; factors for maintenance of financial support; latent variables, nonprofit organizations; structural equation modeling

Due to certain developments in the business of fundraising, there exists an increasing necessity to analyze theoretically and empirically donor behavior. This article examines donor retention for the donor's point of view. It concretizes behavioral antecedents and their relationships among one another. The investigated determinants are commitment, trust, satisfaction and involvement. The empirical analysis shows that all variables have an influence on donor retention, although some of them only indirectly.

\section{Literaturverzeichnis}

Ahearne, Michael, C. B. Bhattacharya und Thomas Gruen (2005), Antecedents and Consequences of CustomerCompany Identification: Expanding the Role of Relationship Marketing, in: Journal of Applied Psychology, 90. Jg., Heft 3, S. 574-585.

Allen, Natalie J. und John P. Meyer (1990), The Measurement and Antecedents of Affective, Continuance and Normative Commitment to the Organization, in: Journal of Occupational Psychology, 63. Jg., Heft 1, S. 1-18.

Andreasen, Alan R. und Philip Kotler (2008), Strategic Marketing for Nonprofit Organizations, 7. Aufl., Upper Saddle River.

Arnett, Dennis B., Steve D. German und Shelby D. Hunt (2003), The Identity Salience Model of Relationship Marketing Success: The Case of Nonprofit Marketing, in: Journal of Marketing, 67. Jg., Heft 2, S. 89-105.

Bagozzi, Richard P. und YoujaeYi (1988), On the Evaluation of Structural Equation Models, in: Journal of the Academy of Marketing Science, 16. Jg., Heft 1, S. 74-94.

Bakay, Zoltan und Manfred Schwaiger (2006), Kundenbindung im liberalisierten Strommarkt, in: Die Betriebswirtschaft, 66. Jg., Heft 3, S. 326-344.

Bennett, Roger (2006), Predicting the Lifetime Durations of Donors to Charities, in: Journal of Nonprofit \& Public Sector Marketing, 15. Jg., Heft 1/2, S. 45-67.

Bennett, Roger und Anna Barkensjo (2005), Causes and consequences of donor perceptions of the quality of the relationship marketing activities of charitable organizations, in: Journal of Targeting, Measurement and Analysis for Marketing, 13. Jg., Heft 2, S. 122-139.

Bennett, Roger und Rehnuma Ali-Choudhury (2009), Second-gift Behavior of First-Time Donors to Charity: An Empirical Study, in: International Journal of Nonprofit and Voluntary Sector Marketing, 14. Jg., Heft 3, S. 161-180.

Bhattacharya, C. B., Rao Hayagreeva und Mary Ann Glynn (1995), Understanding the Bond of Identification: An Investigation of Its Correlates among Art Museum Members, in: Journal of Marketing, 59. Jg., Heft 4, S. 46-57.

Britton, Julie Edel und Josh Rose (2004), Thinking about Relationship Theory, in: Managing Customer Relationships - A Strategic Framework, hrsg. von Don Peppers und Martha Rogers, Hoboken, S. 38-50.

Bruhn, Manfred (1999), Relationship Marketing - Neustrukturierung der klassischen Marketinginstrumente durch eine Orientierung an Kundenbeziehungen, in: Strategisches Management und Marketing, hrsg. von Rudolf Grünig und Martier Pasquier, Bern u. a., S. 189-217.

Bruhn, Manfred (2009), Relationship Marketing: Das Management von Kundenbeziehungen, 2. Aufl., München.

Burgy, Catherine (2008), Nonprofit-Marketing: Messung von Spenderzufriedenheit bei Stiftungen als Fundraisinginstrument, Saarbrücken.

Bussel, Helen und Deborah Forbes (2006), Developing Relationship Marketing in the Voluntary Sector, in: Journal of Nonprofit and Voluntary Sector Marketing, 15. Jg., Heft 1/2, S. 151-174.

Crosby, Lawrence A., Kenneth R. Evans und Deborah Cowles (1990), Relationship Quality in Service Selling: An Interpersonal Influence Perspective, in: Journal of Marketing, 54. Jg., Heft 3, S. 68-81.

Dick, Alan S. und Kunal Basu (1994), Customer Loyalty: Toward an Integrated Conceptual Framework, in: Journal of the Academy of Marketing Science, 22. Jg., Heft 2, S. 99-113.

Doney, Patricia M. und Joseph P. Cannon (1997), An Examination of the Nature of Trust in Buyer-Seller Relationships, in: Journal of Marketing, 61. Jg., Heft 2, S. 35-51.

Eggert, Andreas (1999), Kundenbindung aus Kundensicht: Konzeptualisierung - Operationalisierung - Verhaltenswirksamkeit, Diss., Wiesbaden. 
Eggert, Andreas, Sabrina Helm und Ian Garnefeld (2007), Kundenbindung durch Weiterempfehlung? Eine experimentelle Untersuchung der Wirkung positiver Kundenempfehlungen auf die Bindung des Empfehlenden, in: Marketing ZFP, 29. Jg., Heft 4, S. 233-245.

Esch, Franz-Rudolf, Marco Hardiman und Greg Kiss (2002), Gestaltung von Handelsauftritten im Internet, in: Der Handel im Informationszeitalter: Konzepte - Instrumente - Umsetzung, hrsg. von Dirk Möhlenbruch und Michaela Hartmann, Wiesbaden, S. 227-252.

File, Karen Maru, Ben B. Judd und Russ Alan Price (1996), Perceptions of Quality in the Nonprofit Relationship, in: Journal of Nonprofit \& Public Sector Marketing, 4. Jg., Heft 1/2, S. 75-87.

Fornell, Claes und David F. Larcker (1981), Evaluating Structural Equation Models with Unobservable Variables and Measurement Error, in: Journal of Marketing Research, 18. Jg., Heft 1, S. 39-50.

Garbarino, Ellen und Mark S. Johnson (1999), The Different Roles of Satisfaction, Trust, and Commitment in Customer Relationships, in: Journal of Marketing, 63. Jg., Heft 2, S. 70-87.

Giere, Jens, Bernd Wirtz und Oliver Schilke (2006), Mehrdimensionale Konstrukte, in: Die Betriebswirtschaft, 66. Jg., Heft 6, S. 678-695.

Gordon, Mary Ellen, Kim McKeage und Mark Alexander Fox (1998), Relationship Marketing Effectiveness: The Role of Involvement, in: Psychology \& Marketing, 15. Jg., Heft 5, S. 443-459.

Graeff, Peter (1998), Vertrauen zum Vorgesetzten und zum Unternehmen, Berlin.

Green, Corliss L. und Deborah J. Webb (1997), Factors Influencing Monetary Donations to Charitable Organizations, in: Journal of Nonprofit \& Public Sector Marketing, 5. Jg., Heft 3, S. 19-40.

Gundlach, Gregory. T., Ravi S. Achrol und John T. Mentzer (1995), The Structure of Commitment in Exchange, in: Journal of Marketing, 59. Jg., Heft 1, S. 78-92.

Haibach, Marita (2006), Handbuch Fundraising: Spenden, Sponsoring, Stiftungen in der Praxis, 3. Aufl., Frankfurt am Main.

Helmig, Bernd, Robert Purtschert und Claudio Beccarelli (2006), Erfolgsfaktoren im Nonprofit-Management, in: Nonprofit-Management - Beispiele für Best-Practices im Dritten Sektor, hrsg. von Bernd Helmig und Robert Purtschert, 2. Aufl., Wiesbaden, S. 351-359.

Hermes, Vera (2007), „Das ist ein super Job, den ich hier habe!“, in: Absatzwirtzschaft, 50. Jg., Heft 11, S. 100-103.

Herrmann, Andreas, Frank Huber und Christine Braunstein (2000), Ein Erklärungsansatz der Kundenbindung unter Berücksichtigung der wahrgenommenen Handlungskontrolle, in: Die Betriebswirtschaft, 60. Jg., Heft 3, S. 293-313.

Hofmeyr, Jan und Butch Rice (2002), Commitment Marketing - Markentreue aus Begeisterung, München.

Hohl, Nikolaus A. D. und Julia Naskrent (2009), Involvement - Forschungsstand und Neukonzeption, Arbeitspapier des Lehrstuhls für Marketing, Siegen.

Hohn, Bettina (2001), Internet-Marketing und Fundraising für Nonprofit Organisationen, Diss., Wiesbaden.

Homburg, Christian und Annette Giering (2001), Personal Characteristics as Moderators of the Relationship between Customer Satisfaction and Loyalty: An Empirical Analysis, in: Psychology \& Marketing, 18. Jg., Heft 1, S. 43-66.

Homburg, Christian und Phoebe Kebbel (2001), Involvement als Determinante der Qualitätswahrnehmung von Dienstleistungen, in: Die Betriebswirtschaft, 61. Jg., Heft 1, S. $42-59$.

Hulland, John (1999), Use of Partial Least Squares (PLS) in Strategic Management Research: A Review of four Recent Studies, in: Strategic Management Journal, 20. Jg., Heft 2, S. 195-204.

Jahn, Steffen (2007), Strukturgleichungsmodellierung mit LISREL, AMOS und SmartPLS - Eine Einführung, Arbeitspapier Nr. 86 des Lehrstuhls für Marketing und Handelsbetriebslehre an der Universität Chemnitz, Chemnitz.

Jaritz, Sabine (2008), Kundenbindung und Involvement: Eine empirische Analyse unter besonderer Berücksichtigung von Low Involvement, Diss., Wiesbaden.

Jeker, Karin (2002), Das Bindungsverhalten von Kunden in Geschäftsbeziehungen: Theoretische und empirische Betrachtung der Kundenbindung aus Kundensicht, Bern u. a.

Johnson, Mark S. und Ellen Garbarino (2001), Customers of Performing Arts Organisations: Are Subscribers Different from Nonsubscribers?, in: International Journal of Nonprofit and Voluntary Sector Marketing, 6. Jg., Heft 1, S. 61-77.

Jones, Thomas O. und W. Earl Sasser (1995), Why Satisfied Customers Defect, in: Harvard Business Review, 73. Jg., Heft 6, S. 88-99.

Krafft, Manfred (2007), Kundenbindung und Kundenwert, 2. Aufl., Heidelberg.

Kristoffersen, Line und Sangeta Singh (2004), Successful Application of a Customer Relationship Management Program in a Non-Profit Organization, in: Journal of Marketing Theory and Practice, 12. Jg., Heft 2, S. 28-42.

Lomberg, Carina (2008), Personalanreizstrategien junger Wachstumsunternehmen, Lohmar.

Lüthje, Christian (2008), Der Prozess der Innovation: Das Zusammenwirken von technischen und ökonomischen Akteuren, Habil., Tübingen.

MacMillan, Keith, Kevin Money, Arthur Money und Steve Downing (2005), Relationship Marketing in the Not-forprofit Sector: an Extension and Application of the Commitment-Trust Theory, in: Journal of Business Research, 58. Jg., Heft 6, S. 806-818.

Market, Gunnar (2008), Weiterempfehlung als Marketingziel, Wiesbaden. 
Michaelidou, Nina und Sally Dibb (2008), Consumer Involvement - A New Perspective, in: The Marketing Review, 8. Jg., Heft 1, S. 83-99.

Michalski, Silke und Bernd Helmig (2010), Management von Nonprofit-Beziehungen - ein Service Value Chainorientierter Ansatz, in: Management von Kundenbeziehungen: Perspektiven - Analysen - Strategien - Instrumente, hrsg. von Dominik Georgi und Karsten Hadwich, Wiesbaden, S. 229-249.

Mittal, Banwari (1995), A Comparative Analysis of Four Scales of Consumer Involvement, in: Psychology \& Marketing, 12. Jg., Heft 7, S. 663-682.

Mittal, Banwari und Myung-Soo Lee (1989), A Causal Model of Consumer Involvement, in: Journal of Economic Psychology, 10. Jg., Heft 3, S. 363-389.

Morgan, Robert M. und Shelby D. Hunt (1994), The Commitment-Trust Theory of Relationship Marketing, in: Journal of Marketing, 58. Jg., Heft 3, S. 20-38.

Neumann, Markus M. (2007), Konsumentenvertrauen - Messung, Determinanten und Konsequenzen, Diss., Wiesbaden.

Oliver, Richard L. (1981), Measurement and Evaluation of Satisfaction Process in Retail Setting, in: Journal of Retailing, 57. Jg., Heft 3, S. 25-48.

Peltier, James W. und John A. Schibrowsky (1995), The Direct Marketing of Charitable Causes: a Study of US Fundraisers, in: Journal of Direct Marketing, 9. Jg., Heft 3, S. 72-80.

Ranaweera, Chatura und Jaideep Prabhu (2003), On the Relative Importance of Customer Satisfaction and Trust as Determinants of Customer Retention and Positive Word of Mouth, in: Journal of Targeting, Measurement and Analysis for Marketing, 12. Jg., Heft 1, S. 82-90.

Ringle, Christian M., Sven Wende und Alexander Will (2005), SmartPLS 2.0 (beta), http://www.smartpls.de (Zugriff: 5.7.2008).

Ripperger, Tanja (2003), Ökonomik des Vertrauens: Analyse eines Organisationsprinzips, Diss., Tübingen.

Rothschild, Michael L. (1979), Marketing Communications in Nonbusiness Situations or Why it's so Hard to Sell Brotherhood Like Soap, in: Journal of Marketing, 43. Jg., Heft 2, S. 11-20.

Saab, Samy (2007), Commitment in Geschäftsbeziehungen - Konzeptualisierung und Operationalisierung für das Business-to-Business-Marketing, Diss., Wiesbaden.

Sargeant, Adrian (2001), Managing Donor Defection, in: New Directions for Philantropic Fundraising, 9. Jg., Heft 32 , S. 59-74.

Sargeant, Adrian (2008), Donor Retention: What Do We Know and What Can We Do About It?, http://www.nsfre.org/ content_documents/Donor_Retention_What_Do_We_Know.pdf (Zugriff: 10.1.2009).

Sargeant, Ad̄rian, John B. Ford, und Douglas C. West (2006), Perceptual Determinants of Nonprofit Giving Behaviour, in: Journal of Business Research, 59. Jg., Heft 2, S. 155-165.

Sargeant, Adrian und Lucy Woodliffe (2007), Building Donor Loyalty: The Antecedents and Role of Commitment in the Context of Charity Giving, in: Journal of Nonprofit and Public Sector Marketing, 18. Jg., Heft 2, S. 47-68.

Sargeant, Adrian und Stephen Lee (2002), Individual and Contextual Antecedents of Donor Trust in the Voluntary Sector, in: Journal of Marketing Management, 18. Jg., Heft 7/8, S. 779-802.

Sargeant, Adrian und Stephen Lee (2002 a), Improving Public Trust in the Voluntary Sector: An Empirical Analysis, in: International Journal of Nonprofit and Voluntary Sector Marketing, 7. Jg., Heft 1, S. 68-83.

Sargeant, Adrian und Stephen Lee (2004), Trust and Relationship Commitment in the United Kingdom Voluntary Sector: Determinants of Donor Behavior, in: Psychology and Marketing, 21. Jg., Heft 8, S. 613-635.

Sargeant, Adrian und Stephen Lee (2004 a), Donor Trust and Relationship Commitment in the U.K. Charity Sector: The Impact on Behavior, in: Nonprofit and Voluntary Sector Quarterly, 33. Jg., Heft 2, S. 185-202.

Sauer, Nicole (2003), Consumer Sophistication - Messung, Determinanten und Wirkung auf Kundenzufriedenheit und Kundenloyalität, Diss., Wiesbaden.

Schneider, Willy (1997), Marktsegmentierung im Spendensektor, in: Jahrbuch der Absatz- und Verbrauchsforschung, 43. Jg., Heft 3, S. 254-269.

Shabbir, Haseeb, Dayananda Palihawadana und Des Thwaites (2007), Determining the Antecedents and Consequences of Donor-Perceived Relationship Quality - A Dimensional Qualitative Research Approach, in: Psychology \& Marketing, 24. Jg., Heft 3, S. 271-293.

Sirdeshmukh, Deepak, Jagdip Singh und Barry Sabol (2002), Consumer Trust, Value, and Loyalty in Relational Exchanges, in: Journal of Marketing, 66. Jg., Heft 1, S. 15-37.

Süß, Stefan (2007), Die psychologische Beziehung zwischen Unternehmen und freien Mitarbeitern: Eine empirische Untersuchung des Commitments und der arbeitsbezogenen Erwartungen von IT-Freelancern, Arbeitspapier Nr. 406 des Lehrstuhls für Betriebswirtschaftslehre, insbesondere Organisation und Planung der FernUniversität Hagen, Hagen.

Swanson, Scott R., J. Charlene Davis und Yushan Zhao (2007), Motivations and Relationship Outcomes: The Mediating Role of Trust and Satisfaction, in: Journal of Nonprofit \& Public Sector Marketing, 18. Jg., Heft 2, S. 1-25.

Teichert, Thorsten und Katja Rost (2003), Trust, Involvement Profile and Customer Retention - Modeling, Effects and Implications, in: International Journal of Technology Management, 26. Jg., Heft 5/6, S. 621-639.

Thomas, James L., Brent J. Cunningham und Joann Krauss Williams (2002), The Impact of Involvement, Perceived Moral Intensity, and Satisfaction upon Trust in Non-Profit Relational Contexts: Processes and Outcomes, in: Journal of Nonprofit \& Public Sector Marketing, 10 Jg., Heft 1, S. 93-115. 


\section{Julia Naskrent und Philipp Siebelt}

Tidwell, Michael V. (2005), A Social Identity Model of Prosocial Behaviors within Nonprofit Organizations, in: Nonprofit Management \& Leadership, 15. Jg., Heft 4, S. 449-467.

TNS Infratest (2009), 15 Jahre Deutscher Spendenmonitor - Fakten und Trends im Zeitverlauf, http://www.tnsinfratest.com/presse/pdf/Presse/2009_12_01_15_Jahre_Deut-scher_Spendenmonitor.pdf (Zugriff: 2.12.2009).

Urselmann, Michael (1998), Erfolgsfaktoren im Fundraising von Nonprofit-Organisationen, Diss., Wiesbaden.

Urselmann, Michael (2007), Fundraising. Professionelle Mittelbeschaffung für Nonprofit-Organisationen, 4. Aufl., Bern.

von Stenglin, Ariane (2008), Commitment in der Dienstleistungsbeziehung: Entwicklung eines integrierten Erklärungs- und Wirkungsmodells, Diss., Wiesbaden.

Voss, Kathrin (2007), Öffentlichkeitsarbeit von Nichtregierungsorganisationen, Wiesbaden.

Wilke, Claus (2007), Informationssuche und Konsumentenvertrauen am Beispiel der Versicherungswirtschaft: Eine empirische Untersuchung, Hamburg.

Wünschmann, Stefan und Stefan Müller (2006), Markenvertrauen: Ein Erfolgsfaktor des Markenmanagements, in: Konsumentenvertrauen: Konzepte und Anwendungen für ein nachhaltiges Kundenbindungsmanagement, hrsg. von Hans H. Bauer, Marcus M. Neumann und Anja Schüle, München, S. 221-234.

Zimmer, Annette und Stefan Nährlich (1993), Nonprofit-Management und -Marketing mehr als Betriebsführung und Marktorientierung, in: Zeitschrift für öffentliche und gemeinwirtschaftliche Unternehmen, 16. Jg., Heft 3, S. 345-354. 